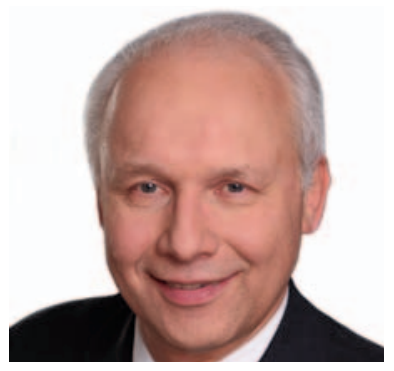

\title{
Denosumab - erste Wahl bei der medikamentösen Therapie von Knochenmetastasen
}

\section{Hartmut Link}

Klinik für Innere Medizin I, Westpfalz-Klinikum Kaiserslautern, Kaiserslautern, Deutschland

Abstractübersetzung aus Chen F, Pu F: Safety of denosumab versus zoledronic acid in patients with bone metastases: a meta-analysis of randomized controlled trials. Oncol Res Treat 2016;39:453-459.

Sicherheit von Denosumab im Vergleich mit Zoledronsäure bei Patienten mit Knochenmetastasen: eine Metaanalyse randomisierter kontrollierter Studien

\section{Schlüsselwörter}

Sicherheit · Denosumab · Zoledronsäure · Knochenmetastasen . Metaanalyse

\section{Zusammenfassung}

Einführung: Knochenmetastasen führen zu lokaler Knochenzerstörung und skelettalen Komplikationen. Bisphosphonate, insbesondere Zoledronsäure (ZS), spielen eine zentrale Rolle bei der Behandlung von Knochenmetastasen. Einige Studien haben gezeigt, dass Denosumab skelettbezogene Ereignisse bei metastasierter Knochenerkrankung möglicherweise effektiver verzögern und verhindern kann als ZS. Daher haben wir die Sicherheit von Denosumab und ZS systematisch überprüft und bewertet.

Methoden: In den Datenbanken PubMed, EMBASE, Cochrane Library, Web of Science with Conference Proceedings, Elsevier und China National Knowledge Infrastructure (CNKI) wurde eine Suche bis einschließlich Oktober 2015 durchgeführt. Zwei un- abhängige Prüfer extrahierten anhand eines Standardprotokolls Daten aus jeder geeigneten Studie; zur Analyse und Bewertung der aus den entsprechenden Artikeln entnommenen Daten wurden sowohl Fixed-Effects- als auch Random-Effects-Modelle herangezogen.

Ergebnisse: In die Auswertung wurden 6 randomisierte kontrollierte Studien mit 13733 Patienten einbezogen. Das Auftreten unerwünschter Ereignisse war im Allgemeinen ähnlich in den Denosumab- und ZS-Gruppen, mit Ausnahme von Anämie und Anorexie bei Patienten mit Knochenmetastasen sowie Rückenund Knochenschmerzen. Jedoch gab es beim Auftreten schwerwiegender unerwünschter Ereignisse wie Hypokalzämie, renale Nebenwirkungen und neue primäre Malignome einen signifikanten Unterschied zwischen den Denosumab- und ZS-Gruppen. Nur beim Auftreten von Osteonekrose des Kiefers zeigte sich kein signifikanter Unterschied zwischen den Denosumab- und ZSGruppen von Patienten mit Knochenmetastasen.

Schlussfolgerung: Bei Patienten mit Knochenmetastasen war Denosumab sicherer bei der Verzögerung bzw. Prävention von skelettbezogenen Ereignissen und verhinderte laut der vorliegenden Metaanalyse im Vergleich zu ZS eine Verschlimmerung der Schmerzen.

C 2017 S. Karger GmbH, Freiburg

\section{KARGER}

Fax +497614520714 information@karger.com www.karger.com

\section{(C) 2017 S. Karger GmbH, Freiburg}




\section{Transfer in die Praxis}

\section{Hintergrund}

Knochenmetastasen treten sehr häufig bei fortgeschrittenen Krebsleiden von Prostata, Mamma, Nieren und Lunge auf, aber auch bei allen sonstigen Malignomen. Daraus resultierende Komplikationen wie Frakturen, Hyperkalzämie, Schmerzen, neurologische Ausfälle u.a. beeinträchtigen die Lebensqualität enorm und können das Überleben der Patienten verkürzen. Die medikamentöse Prophylaxe dieser Komplikationen ist von ihrer Therapie nicht zu unterscheiden.

Es ist daher ein wesentlicher Standard der Supportivtherapie, solche ossären Probleme zu behandeln und zu vermeiden. Bei Patienten mit ossären Tumormanifestationen stehen folgende Therapieoptionen zu Verfügung (aus [1]):

- medikamentöse Schmerztherapie

- lokale Bestrahlung

- operative Intervention

- systemische Tumortherapie

- Zytostatika, Hormontherapie, zielgerichtete Substanzen, immunmodulierende Therapie u.a.

- Radionuklide

- Bisphosphonate bzw. RANK-Ligand-Antikörper.

Patienten mit einer kurzen Lebenserwartung von etwa 3 Monaten müssen nicht mit einer knochenstabilisierenden Therapie behandelt werden [2].

Bisphosphonate, insbesondere Zoledronat (ZA), können skelettale Komplikationen effektiv reduzieren, indem die Osteoklasten inhibiert werden. Die Osteoklasten können auch durch die Neutralisierung des Rank-Liganden mit Denosumab inhibiert werden. Die S3Leitlinie «Supportive Therapie bei onkologischen Patientlnnen» der Deutschen Krebsgesellschaft publizierte eine Metaanalyse mit 31 Vergleichsstudien von Bisphosphonaten mit Placebo mit insgesamt 7823 Patienten. Bisphosphonate reduzierten dabei die Anzahl der skelettal bezogenen Ereignisse (RR 0,83; 95\% KI 0,78-0,88\%). Auch Schmerzen wurde in der Mehrzahl der Studien vermindert [1].

Mehrere Studien zeigten eine stärkere Wirkung und klinische Vorteile von Denosumab im Vergleich zu ZA. Allerdings ist Denosumab beim Multiplen Myelom noch nicht zugelassen. Die S3-Leitlinie zur Supportivtherapie formuliert folgende evidenzbasierte Empfehlung [1]:

- Zur osteoprotektiven Therapie stehen Denosumab und Bisphosphonate zur Verfügung. Bei Patienten mit ossären Metastasen bei Mammakarzinom bzw. Prostatakarzinom führt die Gabe von Denosumab gegenüber ZA zu einer zahlenmäßig geringen, statistisch signifikanten Reduktion der skelettbezogenen Ereignisse.

- Bei anderen Outcome-Parametern (wie z.B. Schmerz, Lebensqualität, Rückenmarkkompression, Mortalität, Kieferosteonekrose) besteht keine Evidenz für einen Unterschied.
- Daten zum Vergleich von Denosumab gegenüber den anderen Bisphosphonaten liegen nicht vor. Die Daten zum Vergleich von Denosumab und Bisphosphonaten bei anderen soliden Tumoren sind in Bezug auf den Endpunkt skelettbezogener Ereignisse unzureichend.

Sowohl mit ZA als auch mit Denosumab besteht das geringe Risiko der Kieferosteonekrose, das durch eine prätherapeutische Sanierung von entzündlichen oder infektiösen Erkrankungen von Zähnen, Paradontium und Kieferknochen und durch regelmäßige zahnärztliche Untersuchungen minimiert werden kann. Außerdem ist wichtig, dass die Patienten eine regelmäßige und sorgfältige Mundhygiene durchführen. Es gibt mehrere Studien, die zeigen, dass nach dem ersten Jahr mit monatlichen ZA-Infusionen dreimonatliche Intervalle möglich sind. Für Denosumab liegen dazu noch keine ausreichenden Daten vor.

\section{Fazit für die Praxis}

Die vorliegende Metaanalyse von Chen und Pu bestätigt, dass Denosumab im Vergleich zu ZA Skelettkomplikationen effektiver vermindert und z.B. beim Mammakarzinom die Lebensqualität erhöht. Auch die Notwendigkeit der palliativen Strahlentherapie von Knochenmetastasen ist mit Denosumab geringer. Neben der fehlenden Nephrotoxizität bietet Denosumab deutliche Vorteile gegenüber ZA und sollte die erste Wahl der medikamentösen Therapie von Knochenmetastasen sein. Zu beachten ist die orale Substitution von mindestens 500 mg Calcium und 400 I.E. Vitamin D pro Tag, um Hypokalzämien zu vermeiden.

\section{Disclosure Statement}

Hiermit erkläre ich, dass folgende Interessenskonflikte bestehen: Berufstätigkeit: Amgen, Grifols, MSD, Novartis-Sandoz-Hexal, Teva, Viforpharma; Vorträge: Amgen, Novartis-Sandoz-Hexal, MSD, Teva, Viforpharma; Finanzierung wissenschaftlicher Untersuchungen: Amgen, MSD, Teva.

\section{Literatur}

1 Leitlinienprogramm Onkologie: S3-Leitlinie Supportive Therapie bei onkologischen PatientInnen. http://leitlinienprogramm-onkologie.de/SupportiveTherapie.95.0.html (Zugriff 22.01.2017).

2 Coleman R, Body JJ, Aapro M, Hadji P, Herrstedt J, on behalf of the EGWG: Bone health in cancer patients: ESMO Clinical Practice Guidelines. Ann Oncol 2014;25:iii124-iii137.

Kontaktadresse: Prof. Dr. Hartmut Link, Klinik für Innere Medizin I, Westpfalz-Klinikum Kaiserslautern, Hellmut-Hartert-Straße 1, 67653 Kaiserslautern, Deutschland, hlink@kabelmail.de 